\title{
Télécommande et automatisme dans les usines hydro-électriques
}

\author{
Jean-Marc Fermé \\ GEC Alsthom \\ Division Neyrpic
}

\section{Introduction}

Les régulateurs de vitesse sont partie prenante dans les changements liés à l'évolution de l'automatisation des centrales. Tout en améliorant continuellement les performances de base de ces équipements, de nouvelles fonctionnalités et de nouveaux services ont pu être associés à ces réalisations. Parallèlement les appareils industriels ont profité des progrès technologiques, notamment ceux de la micro-électronique. C'est le cas des communications numé- riques qui permettent aujourd'hui de faire partager des informations riches à d'autres applications. Les régulateurs DIGIPID de NEYRPIC participent à ces changements.

Après un bref passage sur les améliorations apportées aux fonctionnalités de base, il est détaillé les nouveaux services apportés par l'atelier de mise au point et de surveillance STATUS : assistance à la mise en service, mesures continues en opération, aide à la maintenance et téléinformation.

\section{Remote control and automatisation of hydro power plants}

Speed governors are a key element in the automatisation progressing of hydro power plants. New functions and new services have been linked to these achievements, while continuously improving basic performances of speed governors.

Besides, industrial equipment took a large advantage from technological progress, namely from microelectronics. This applies to digital communications which enables today the sharing of rich informations to other applications. Neyrpic DIGIPID speed governors are progressing the same way. After a brief description of these basic improvments, new services are described, giving some informations on the following STATUS setting and monitoring functions: aided setting, continuous measures while operating the unit, aided maintenance and remote monitoring. 


\section{SYSTEME GLOBAL DIGIPID}

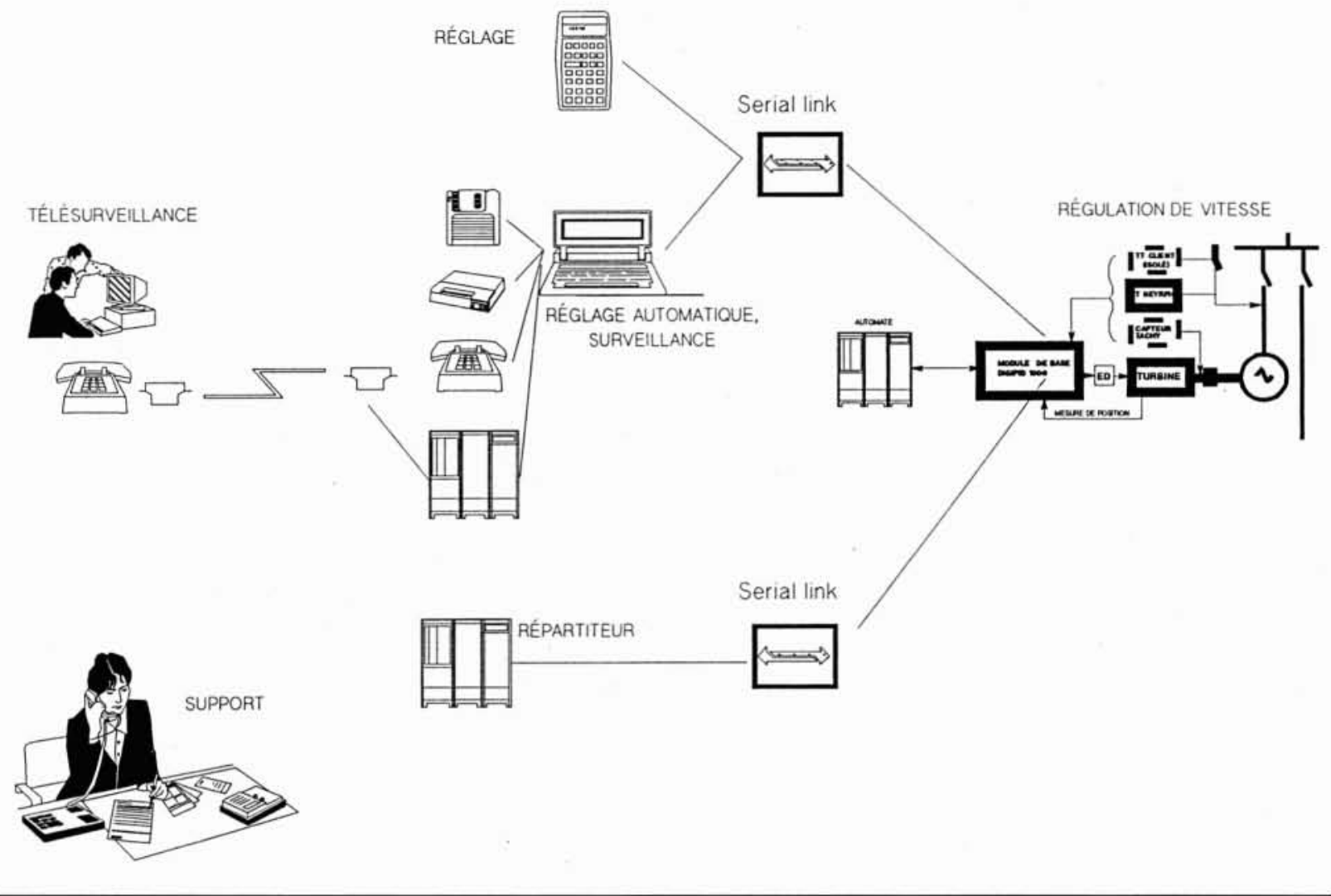

\section{Evolution des fonctions de base}

Cette progression continue provient de l'influence de multiples acteurs dont les principaux sont les utilisateurs, les ingénieurs conseils, les commissions de normalisation internationales, les constructeurs de machines. Nous citons ci-après quelques exemples simples, qui ne sont en soit pas très spectaculaires.

Du point de vue externe à l'équipement, la contribution des techniques numériques à l'amélioration de la régulation de vitesse a été importante pour la réduction, à faible coût, de dérives de réglages sous l'influence du temps ou de la température.

Cela est particulièrement remarquable pour la fonction de mesure de vitesse/fréquence, qui depuis l'origine est réalisée de manière totalement numérique pour tous les régulateurs de vitesse DIGIPID 02 et DIGIPID 1000. Cette amélioration a une incidence directe d'une part sur la précision de la répartition de charge entre plusieurs groupes producteurs sous la seule dépendance du statisme permanent, et d'autre part sur la réduction importante des contrôles périodiques de recalibrage du régulateur.

Par ailleurs des efforts de développement importants en traitement du signal ont permis d'amener l'information vitesse groupe, mesurée sur roue dentée, à un niveau de qualité comparable à celui obtenu par mesure sur les TT ; cette possibilité est aujourd'hui disponible pour la plupart des machines à arbre horizontal. Ainsi, la régulation de vitesse possède une souplesse complémentaire d'adaptation aux spécifications particulières de certains clients internationaux.

Les machines hydrauliques ont-elles aussi évolué, appelant au traitement de nouvelles fonctions à l'intérieur du régulateur. En général, et dans un but de simplification de l'automatisme général et de l'exploitation du groupe, ceci ne transparaît donc pas à l'extérieur de l'équipement de régulation. Par conséquent, on peut dire avec certitude que la turbine et la régulation sont aujourd'hui liées plus étroitement que par le passé, notamment pour les machines à organes réglants multiples.

\section{Les DIGIPID et leur environnement}

NEYRPIC s'est engagé de manière très volontariste vers l'ouverture de la régulation de vitesse vers l'environnement d'exploitation et de surveillance des machines en dotant ses régulateurs numériques DIGIPID d'un puissant lien de communication externe. Le schéma 1 présente l'extension des principaux services accessibles, tout en rappelant qu'à la base l'ensemble des fonctions de régulation de vitesse restent en permanence parfaitement remplies.

L'ensemble des services de réglage et de surveillance sont accessibles via un équipement indépendant et externe au régulateur de vitesse. Chaque utilisateur peut donc optimiser les ressources de son application soit en installant un équipement fixe et permanent, soit en utilisant un outillage 
mobile et temporaire. L'atelier de mise au point et de surveillance STATUS a été développé pour la grande famille des compatibles PC/PS, famille dans laquelle le choix de matériel est suffisamment large, d'autant que certaines centrales disposent déjà d'un calculateur de ce type.

NEYRPIC a cependant maintenu pour les régulateurs DIGIPID l'utilisation alternative d'un terminal léger et économique, fixe pour le DIGIPID 02 et mobile/fixe pour le DIGIPID 1000. Il donne accès à l'ajustage des paramètres de fonctionnement du régulateur.

\section{STATUS et le réglage des régulateurs}

Le coût et la qualité de la mise en service d'un équipement sont certainement liés à la performance et des outillages utilisés et des mesures effectuées. Lors des premières réalisations industrielles de régulateurs numériques, il est clairement apparu une importante difficulté d'observation de ce qui se passait précisément à l'intérieur de l'équipement en fonctionnement. Dans les générations de régulateurs précédentes, les comparateurs, voltmètres, oscilloscopes, enregistreurs analogiques ont été des outils de mise au point essentiels. On peut encore se rappeler avec nostalgie les heures passées pour le calibrage des instruments, le raccordement en fils volants sur les points de mesure, et pour les moins expérimentés les perturbations des mesures et les parasites introduits dans le régulateur par ces sondes.

La nécessité d'améliorer cette situation - régulateur numérique obscur et délicatesse des mesures analogiques - a conduit NEYRPIC à développer une solution qui ne présenterait pas ces inconvénients. Lors du développement du DIGIPID 1000 en 1988, les concepteurs ont donc été amenés à donner une forte contribution technique sur ce sujet. La popularité croissante des compatibles PC/PS ne pouvait que faciliter l'orientation du projet : un système où les mesures directes et pures seraient prises en charge par le régulateur de vitesse puis transmises en temps réel sur une liaison série vers un calculateur PC/PS externe chargé de leur exploitation. Cela a donc guidé l'organisation du développement, d'une part les applications régulateur et communication, et d'autre part le logiciel d'exploitation sur $\mathrm{PC} / \mathrm{PS}$.

Le DIGIPID 02 ayant été développé au début des années 80 , la connexion avec STATUS est disponible depuis peu, moyennant l'adjonction d'une UC supplémentaire dédiée à la transmission des informations entre le cœur du régulateur et le monde extérieur.

Il est intéressant de développer les fonctionnalités liées au réglage, à la mesure, à la surveillance.

\section{Les mesures et le réglage sur le terrain}

Tout d'abord, STATUS donne accès à la visualisation dynamique des états internes du régulateurs, aussi bien en terme d'états logiques qu'en terme d'informations de régulation telles que la vitesse/fréquence instantanée du groupe en fonctionnement, l'ouverture de l'organe réglant, la commande de l'actionneur, le courant dans l'actionneur, la sortie de tel correcteur, etc. L'écran du PC/PS fonctionne alors comme celui d'un oscilloscope numérique à trois voies, le balayage horizontal étant fonction du temps. Les fonctions de calibrage accessibles au clavier permettent aussi de sélectionner les grandeurs à observer, de geler l'image sur l'écran. Le balayage XY est disponible lorsque l'on n'utilise que deux voies à l'écran.

Les signaux ainsi recueillis peuvent être stockés simultanément sur l'unité de disquette ou le disque dur de l'équipement de supervision PC/PS. Avec les portables du marché actuel, on peut donc stocker l'intégralité de l'information sur une durée d'une centaine d'heures.

Lors de la mise en service de l'équipement, il faut aussi apporter à l'opérateur les moyens d'ajuster ses paramètres. En général, une partie du système est déjà pré-réglée chez le constructeur, et les paramètres de stabilité pour le réseau isolé ont été calculés à l'avance; reste à affiner certains réglages de terrain.

A cet usage, quatre fenêtres de réglage ont été placées sur l'écran de visualisation. L'opérateur sélectionne dans chaque fenêtre le paramètre sur lequel il souhaite agir. La visualisation en temps réel des effets de la modification d'un paramètre aide à réduire la durée d'une optimisation.

Des fonctions statistiques sont venues compléter les réponses temporelles du régulateur. Les informations transmises au PC/PS sont classées selon leur fréquence d'apparition ; ces fonctions de répartition sont un indicateur de synthèse supplémentaire pour la confirmation de la qualité d'un réglage, par comparaison directe avec un gabarit normal.

A l'heure actuelle, hormis l'étude de stabilité, ces optimisations sont conduites par l'opérateur, sur sa seule expérience. NEYRPIC a testé en 89 la faisabilité technique d'une assistance au réglage de certaines fonctions sur le terrain, telle le positionneur d'organe réglant. L'intégration de cette assistance est prévue dans les mois prochains. Et encore une fois, compte tenu de la grande sensibilité des algorithmes d'identification, c'est grâce à la pureté des mesures transmises que l'on peut en attendre de bons résultats.

La première application de l'atelier de mise au point STATUS a été la qualification du matériel et du logiciel du DIGIPID 1000. Les concepteurs ont ainsi pu mieux garantir les performances de ce régulateur, dans ses moindres détails. Exemple : en visualisant la charge de l'unité centrale, l'analyse de la valeur des traitements numériques a pu être conduite rationnellement.

Une autre application est la connexion via le réseau téléphonique d'un régulateur en opération et de l'autre côté d'un observateur équipé d'un PC/PS. L'échange a lieu en temps réel, avec une dégradation certaine de dynamique pour les modem à 2400 bauds. En cas de besoin, les experts auront accès à distance, plus rapidement et plus précisément, aux informations essentielles. Bien que l'intérêt soit limité en France où les distances sont moyennement élevées, ce système trouvera une place importante pour les autres pays dans l'exploitation temps réel du retour d'expérience. 


\section{Les régulateurs et la surveillance}

L'intérêt grandissant des fonctions de visualisation et de mise au point de STATUS s'est aussi concrétisé par l'intégration dans le régulateur de fonctions élémentaires de surveillance de la régulation de vitesse d'une machine.

Une horloge a été intégrée dans les DIGIPID 1000, afin de donner accès aux fonctions de datation locale d'événements; sa mise à l'heure et les comptes rendus sont accessibles par le lien de communication externe.

D'autre part, le régulateur de vitesse possède certaines informations de haute qualité dynamique, acquises ou calculées, et il est bien placé à ce titre pour effectuer en permanence des traitements locaux. En effet, dans l'hypothèse d'un réseau de communication, il ne semble pas réaliste d'encombrer la ligne par la transmission de grands volumes des données brutes.

Par ailleurs, la solution pour ce nouveau type de traitement devait être économique, le DIGIPID 1000 étant un régulateur de début de gamme. Le compromis qui a été retenu par NEYRPIC est de synthétiser l'information et de conserver les résultats en mémoire non volatile. Cette petite boîte noire calcule en permanence et enregistre les fonctions de répartition ou bien des écarts de fréquence d'un groupe en production sur un réseau, image essentielle et caractéristique lorsqu'il ne s'agit pas d'un grand réseau, ou bien les ouvertures d'organe réglant donnant ainsi une information de base pour corréler ouverture et usure de machine. Ces informations compressées peuvent facilement être transmises vers un équipement de supervision externe.

En conclusion, les régulateurs numériques de vitesse d'aujourd'hui ont évolué vers une prise en compte des fonctions de base plus étendue, et ils englobent maintenant un savoir faire plus important sur le fonctionnement des machines hydrauliques. Ils effectuent un traitement rapproché d'expertise sur la régulation de vitesse. Dans un avenir proche, ils seront amenés à partager ces informations avec d'autres systèmes. Cela pourra se faire d'autant plus rapidement que les utilisateurs s'engageront sur une structure globale à base de réseaux locaux. 